\title{
Main Effects Screening: A Distributed Continuous Quality Assurance Process for Monitoring Performance Degradation in Evolving Software Systems
}

\author{
Cemal Yilmaz ${ }^{\dagger}$, Arvind S. Krishna ${ }^{\ddagger}$, Atif Memon ${ }^{\dagger}$, Adam Porter ${ }^{\dagger}$, Douglas C. Schmidt ${ }^{\ddagger}$, \\ Aniruddha Gokhale ${ }^{\ddagger}$, Balachandran Natarajan ${ }^{\ddagger}$ \\ ${ }^{\dagger}$ Dept. of Computer Science, University of Maryland, College Park, MD 20742 \\ ${ }_{\ddagger}^{\ddagger}$ Dept. of Electrical Engineering and Computer Science, Vanderbilt University, Nashville, TN 37235
}

\begin{abstract}
Developers of highly configurable performanceintensive software systems often use a type of in-house performance-oriented "regression testing" to ensure that their modifications have not adversely affected their software's performance across its large configuration space. Unfortunately, time and resource constraints often limit developers to in-house testing of a small number of configurations and unreliable extrapolation from these results to the entire configuration space, which allows many performance bottlenecks and sources of QoS degradation to escape detection until systems are fielded. To improve performance assessment of evolving systems across large configuration spaces, we have developed a distributed continuous quality assurance (DCQA) process called main effects screening that uses in-the-field resources to execute formally designed experiments to help reduce the configuration space, thereby allowing developers to perform more targeted in-house QA. We have evaluated this process via several feasibility studies on several large, widely-used performance-intensive software systems. Our results indicate that main effects screening can detect key sources of performance degradation in large-scale systems with significantly less effort than conventional techniques.
\end{abstract}

\section{Introduction}

The quality of performance-intensive software systems, such as high-performance scientific computing systems and distributed real-time and embedded (DRE) systems, depend heavily on their infrastructure platforms, such as the hardware, operating system, middleware, and language processing tools. Developers of such systems often need to tune the infrastructure and their software applications to accommodate the (often changing) platform environments and performance requirements. This tuning is commonly done by (re)adjusting a large set (10's-100's) of compile- and runtime configuration options that record and control variable software parameters, such as different operating systems, resource management strategies, middleware and application feature sets; compiler flags; and/or run-time optimization settings. For example, SQL Server 7.0 has 47 configuration options, Oracle 9 has 211 initialization parameters, and Apache HTTP Server Version 1.3 has 85 core configuration options.
Although these software parameters promote flexibility and portability, they also require that the software be tested in an enormous number of configurations. This creates serious challenges for developers who must ensure that their decisions, additions, and modifications work across this large (and often changing) configuration space:

- Settings that maximize performance for a particular platform/context may not be suitable for different ones and certain groups of option settings may be semantically invalid due to subtle dependencies between options.

- Limited QA budgets and rapidly changing code bases mean that developers' QA efforts are often limited to just a few software configurations, forcing them to extrapolate their findings to the entire configuration space.

- The configurations that are tested are often selected in an ad hoc manner, so quality is not evaluated systematically and many quality problems escape detection until systems are fielded.

Since exhaustively testing all configurations is infeasible under the circumstances listed above, developers need a quick way to estimate how their changes and decisions affect software performance across the entire configuration space. To do this, we have developed and evaluated a new hybrid (i.e., partially in-the-field and partially in-house) distributed continuous quality assurance (DCQA) process that improves software quality iteratively, opportunistically, and efficiently by executing QA tasks continuously across a grid of computing resources provided by end-users and distributed development teams.

In prior work [12], we created a prototype DCQA support environment called Skoll that helps developers create, execute, and analyze their own DCQA processes, as described in Section 2. To make it easier to implement DCQA processes, we also integrated model-based software development tools with Skoll, which help developers capture the variant and invariant parts of DCQA processes and the software systems they are applied to within highlevel models that can be processed to automatically generate configuration files and other supporting code artifacts [7]. Some model-based tools integrated with Skoll include the Options Configuration Modeling language (OCML) [14] 
that models configuration options and inter-option constraints and the Benchmark Generation Modeling Language (BGML) [8] that composes benchmarking experiments to observe QoS behavior under different configurations and workloads.

This paper extends our earlier work by developing a new model-based hybrid DCQA process that leverages the extensive (albeit less dedicated) in-the-field computing resources provided by the Skoll grid, weeding out unimportant options to reduce the configuration space, thereby allowing developers to perform more targeted QA using their very limited (but dedicated) in-house resources. This hybrid DCQA process first runs formally designed experiments across the Skoll grid to identify a subset of important performance-related configuration options. Whenever the software changes thereafter, this process exhaustively explores all configurations of the important options using in-house computing resources to estimate system performance across the entire configuration space. This hybrid approach is feasible because the new configuration space is much smaller than the original, and hence more tractable using in-house resources.

This paper presents an evaluation of our new hybrid DCQA process on ACE, TAO, and CIAO (deuce. doc. wustl.edu/Download.html), which are widelyused production quality, performance-intensive middleware frameworks. Our results indicate that (1) hybrid modelbased DCQA tools and processes can correctly identify the subset of options that are important to system performance, (2) monitoring only these selected options helps to quickly detect key sources of performance degradation at an acceptable level of effort, and (3) alternative strategies with equivalent effort give less reliable results.

\section{The Model-based Skoll DCQA Environment}

To improve the quality of performance-intensive software across large configuration spaces, we are exploring distributed continuous quality assurance (DCQA) processes [12] that evaluate various software qualities, such as portability, performance characteristics, and functional correctness, "around-the-world, around-the-clock." To accomplish this, DCQA processes are divided into multiple subtasks, such as running regression tests on a particular system configuration, evaluating system response time under different input workloads, or measuring usage errors for a system with several alternative GUI designs. As illustrated in Figure 1 , these subtasks are then intelligently and continuously distributed to - and executed by - clients across a grid of computing resources contributed largely by end-users and distributed development teams. The results of these evaluations are returned to servers at central collection sites, where they are fused together to guide subsequent iterations of the DCQA processes.

To support this effort we have developed Skoll, a model-

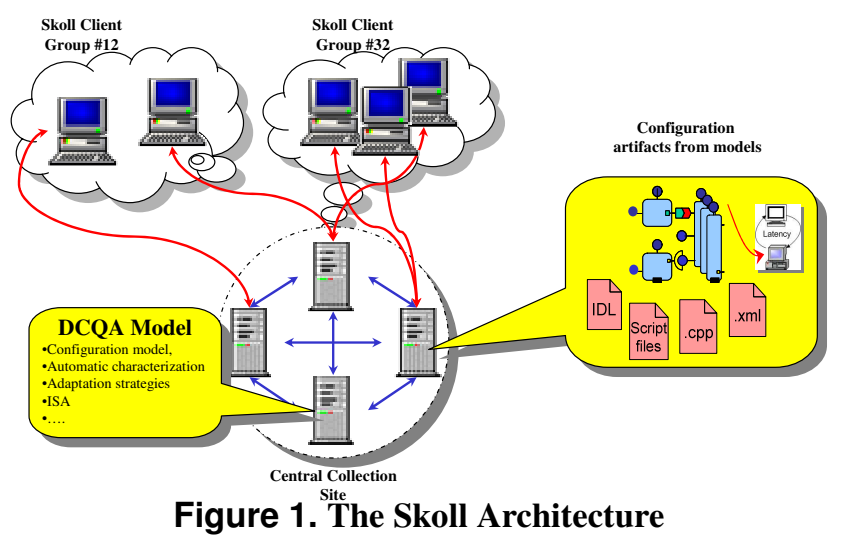

based DCQA environment described at WWW. cs. umd. edu/projects/skoll. For completeness, this section describes some of Skoll's components and services, which include languages for modeling system configurations and their constraints, algorithms for scheduling and remotely executing tasks, and planning technology that analyzes subtask results and adapts the DCQA process in real time.

The cornerstone of Skoll is its formal model of a DCQA process's configuration space, which captures different configuration options and their settings. Since in practice not all combinations of options make sense (e.g., feature X may not be supported on operating system Y), we define inter-option constraints that limit the setting of one option based on the settings of others. A valid configuration is one that violates no inter-option constraints (for the feasibility study in Section 4, we used the OCML modeling tool to visually define the configuration model and to generate the low-level formats used by other Skoll components). Skoll uses this configuration space model to help plan global QA processes, adapt these processes dynamically, and aid in analyzing and interpreting results from various types of functional and performance regression tests.

Since the configuration spaces of performance-intensive software can be quite large, Skoll has an Intelligent Steering Agent (ISA) that uses AI planning techniques to control DCQA processes by deciding which valid configuration to allocate to each incoming Skoll client request. When a client is available, the ISA decides which subtask to assign it by considering many factors, including (1) the configuration model, which characterizes the subtasks that can legally be assigned, (2) the results of previous subtasks, which capture what tasks have already been done and whether the results were successful, (3) global process goals, such as testing popular configurations more than rarely used ones or testing recently changed features more heavily than unchanged features, and (4) client characteristics and preferences, e.g., the selected configuration must be compatible with the OS running on the client machine or configurations must run with user-level - rather than superuser-level - protection modes. 
After a valid configuration is chosen, the ISA packages the corresponding QA subtask into a job configuration, which consists of the code artifacts, configuration parameters, build instructions, and QA-specific code (e.g., developer-supplied regression/performance tests) associated with a software project. Each job configuration is then sent to a Skoll client, which executes the job configuration and returns the results to the ISA (for the feasibility studies described in Section 4, we used the BGML modeling tools [9] to generate most of the code that comprises a job configuration). The ISA can learn from the results and adapt the process, e.g., if some configurations fail to work properly, developers may either want to pinpoint the source of the problems or refocus on other unexplored parts of the configuration space. To control the ISA, Skoll DCQA process designers can develop customized adaptation strategies that monitor the global process state, analyze it, and use the information to modify future subtask assignments in ways that improve process performance.

Since DCQA processes can be complex, Skoll users often need help to interpret and leverage process results. Skoll therefore supports a variety of pluggable analysis tools, such as Classification Tree Analysis (CTA) [1]. In previous work $[12,16]$, for example, we used CTA to diagnose options and settings that were the likely causes of specific test failures. For this work, we developed statistical tools to analyze data from the formally-designed experiments described in the following section.

\section{Performance-Oriented Regression Testing}

As software systems change, developers often run regression tests to detect unintended functional side effects. Developers of performance-intensive systems must also be wary of unintended side effects on their end-to-end QoS. To detect such performance problems, developers often run benchmarking regression tests periodically. As described in Section 1, however, in-house QA efforts can be confounded by the enormous configuration space of highly configurable performance intensive systems, where time and resource constraints (and often high change frequencies) severely limit the number of configurations that can be examined. For example, our earlier experience with applying Skoll to the ACE+TAO middleware [12] found that only a small number of default configurations are benchmarked routinely by the core ACE+TAO development team, who thus get a very limited view of their middleware's QoS. Problems not readily seen in these default configurations therefore often escape detection until systems based on ACE+TAO are fielded by end-users.

This section describes how we address this problem by using the model-based Skoll environment (Section 2) to develop and implement a new hybrid DCQA process called main effects screening. We also describe the formal foundations of our approach, which is based on design of ex- periments theory, and give an example that illustrates key aspects of our approach.

\subsection{The Main Effects Screening Process}

Main effects screening is a technique for rapidly detecting performance degradation across a large configuration space as a result of system changes. Our approach relies on a class of experimental designs called screening designs [15], which are highly economical and can reveal important low order effects (such as individual option settings and option pairs/triples) that strongly affect performance. We call these most influential option settings "main effects."

At a high level, main effects screening involves the following steps: (1) compute a formal experimental design based on the system's configuration model, (2) execute that experimental design across fielded computing resources in the Skoll DCQA grid by running and measuring benchmarks on specific configurations dictated by the experimental design devised in step 1, (3) collect, analyze and display the data so that developers can identify the main effects, (4) estimate overall performance whenever the software changes by evaluating all combinations of the main effects (while defaulting or randomizing all other options), and (5) recalibrate the main effects options by restarting the process periodically since the main effects can change over time, depending on how fast the system changes.

The assumption behind this five step process is that since main effects options are the ones that affect performance most, evaluating all combinations of these option settings (which we call the "screening suite") can reasonably estimate performance across the entire configuration space. If this assumption is true, testing the screening suite should provide much the same information as testing the entire configuration space, but at a fraction of the time and effort since it is much smaller than the entire configuration space. 3.2. Technical Foundations of Screening Designs

For main effects screening to work we need to identify the main effects, i.e., the subset of options whose settings account for a large portion of performance variation across the system's configuration space. One obvious approach is to test every configuration exhaustively. Since exhaustive testing is infeasible for large-scale, highly configurable performance-intensive software systems, however, developers often do some kind of random or ad hoc sampling based on their knowledge of the system. Since our experience indicates that these approaches can be unreliable $[12,8]$, we need an approach that samples the configuration space, yet produces reasonably precise and reliable estimates of overall performance.

The approach we chose for this paper uses formallydesigned experiments, called screening designs, that are highly economical and whose primary purpose is to identify important low-order effects, i.e., first-, second-, or thirdorder effects, where an $n^{\text {th }}$-order effect is an effect caused by the simultaneous interaction of $n$ factors. For instance, 
for certain web server applications, a $1^{\text {st }}$-order effect might be that performance slows considerably when logging is turned on and another might be that it also slows when few server threads are used. A $2^{\text {nd }}$ order effect involves the interaction of two options, e.g., web server performance may slow down when caching is turned off and the server performs blocking reads.

There are many ways to compute screening designs. The one we use is based on traditional factorial designs. Consider a full factorial design involving $k$ binary factors. Such a design exhaustively tests all combinations of the factors. Therefore, the design's run size (number of experimental observations) is $2^{k}$. Although this quickly becomes expensive, it does allow one to compute all effects.

To reduce costs statisticians have developed fractional factorial designs. These designs use only a carefully selected fraction (such as $1 / 2$ or $1 / 4$ ) of a full factorial design. This saves money, but does so by giving up the ability to measure some higher-order effects. This is because the way observations are chosen aliases the effects of some lower-order interactions with some higher-order ones. That is, it lumps together certain high- and low-order effects on the assumption that the high-order effects are negligible.

Screening designs push this tradeoff to an extreme. Roughly speaking, at their smallest, screening designs require only as many observations as the number of effects one wishes to calculate (i.e., $k$ observations to compute $k$, $1^{\text {st }}$-order effects). Of course, experimenters will often use more than the minimum number of observations to improve precision or to deal with noisy processes. As before, this is only possible because the design aliases some low-order with some high-order effects.

\subsection{Screening Designs in Action}

To show how screening designs are computed, we now present a hypothetical example of a software system with 4 binary configuration options, A through D, with no interoption constraints. To compute a specific screening design, developers must (a) decide how many observations they can afford, (b) determine which effects they want to analyze, and (c) select an aliasing strategy consistent with these constraints. Note that in practice screening designs are usually computed by automated tools.

The configuration space for our sample system has $2^{4}=$ 16 configurations. Therefore, the corresponding full factorial design involves 16 observations. Let's assume that our developers, however, can afford to run only 8 observations. Obviously, the full factorial design would therefore be unacceptable. Let's also assume that our developers are mostly interested in capturing the $4,1^{\text {st }}$-order effects (i.e., the effect of each option by itself.) Given this goal the developers decide to use a screening design.

The first step in computing the screening design is to create a $2^{3}$ full factorial design over 3 (arbitrarily selected) options, in this case A, B, and C. This design is shown in Ta- ble 1(a) with the binary option settings encoded as $(-)$ or $(+)$. This is the starting point because $2^{3}=8$ is the maximum number of observations the developers can afford to run.

\begin{tabular}{|c|c|c|}
\hline $\mathrm{A}$ & $\mathrm{B}$ & $\mathrm{C}$ \\
\hline- & - & - \\
+ & - & - \\
- & + & - \\
+ & + & - \\
- & - & + \\
+ & - & + \\
- & + & + \\
+ & + & + \\
\hline
\end{tabular}

(a)

\begin{tabular}{|c|c|c|c|}
\hline $\mathrm{A}$ & $\mathrm{B}$ & $\mathrm{C}$ & $\mathrm{D}$ \\
\hline- & - & - & - \\
+ & - & - & + \\
- & + & - & + \\
+ & + & - & - \\
- & - & + & + \\
+ & - & + & - \\
- & + & + & - \\
+ & + & + & + \\
\hline
\end{tabular}

(b)

\section{Table 1. (a) $2^{3}$ Design and (b) $2_{I V}^{4-1}$ Design}

This initial design would be fine for 3 options. But it can't handle our developer's 4-option system since they've already decided that the full factorial design would be too expensive. We need a way to stretch the current 3-option design to cover the $4^{\text {th }}$ option. As mentioned previously, this is done by aliasing some effects.

Here the developers must specify which effects can be safely aliased. They do this by choosing a desired resolution for the screening design. In resolution $R$ designs, no effects involving $i$ factors are aliased with effects involving less than $R-i$ factors. Since our developers are only interested in computing $1^{\text {st }}$-order effects, they choose a resolution IV design. With this design, $1^{s t}$-order effects will be aliased with $3^{r d}$-order or higher effects and $2^{n d}$-order effects will be aliased with $2^{\text {nd }}$-order or higher effects. Our developers, therefore, are assuming that $3^{r d}$-order or higher effects are negligible and that they are not interested in computing the $2^{\text {nd }}$-order effects. If the developers are unwilling to make these assumptions, then screening designs may be inappropriate for them (or they may need to use the screening designs in an iterative, exploratory fashion).

The final step in the process to compute the specific values of option D consistent with the developers desire for a resolution IV design. To do this a function called a design generator must be determined. This function computes the setting of option $\mathrm{D}$ based on the values of options $\mathrm{A}, \mathrm{B}$ and $\mathrm{C}$. In our hypothetical example, automated tools chose $D=A B C$ as the design generator. This means that for each observation, D's setting is computed by multiplying the settings for options $\mathrm{A}, \mathrm{B}$, and $\mathrm{C}$ (think of + as 1 and - as -1$)$. In general there is no closedform solution for choosing a design generator. In fact, in some situations, none may exist. For this paper we identified design generators using the factex function of the SAS QC package (See http: / / www . math.wpi . edu / saspdf / qc/chap14.pdf). This search-based function 
essentially looks through numerous possible design generators until an acceptable one is found (or until the algorithm gives up, if none can be found).

Table 1(b) gives the final design, which is identified uniquely as a $2_{I V}^{4-1}$ design with the design generator $D=$ $A B C$. The $2^{4-1}$ designation means that the total number of options is 4 , that we will examine a $1 / 2\left(2^{-1}=1 / 2\right)$ fraction of the full factorial design, that the design is a resolution IV screening design, and that the aliasing pattern is $D=A B C$.

After defining the screening design, developers can execute it across the Skoll grid. For our process, each observation involves measuring a developer-supplied benchmarking regression test while the system runs in a particular configuration. Once the data is collected we would analyze it to calculate the effects. For binary options (with settings - or + ), the main effect of option $\mathrm{A}, \mathrm{ME}(\mathrm{A})$, is

$$
M E(A)=z(A-)-z(A+)
$$

where $\mathrm{z}(\mathrm{A}-)$ and $\mathrm{z}(\mathrm{A}+)$ are the mean values of the observed data over all runs where option $\mathrm{A}$ is (-) and where option $\mathrm{A}$ is $(+)$, respectively.

If appropriate, $2^{\text {nd }}$-order effects can be calculated in a similar way. The interaction effect of option A and B, $\operatorname{INT}(\mathrm{A}, \mathrm{B})$ is:

$$
\begin{aligned}
I N T(A, B) & =1 / 2\{M E(B \mid A+)-M E(B \mid A-)\} \\
& =1 / 2\{M E(A \mid B+)-M E(A \mid B-)\}
\end{aligned}
$$

Here $M E(B \mid A+)$ is called the conditional main effect of $\mathrm{B}$ at the + level of A. The effect of one factor (e.g., B) therefore depends on the level of the other factor (e.g., A). Similar equations exist for higher order effects.

Once the effects are computed developers will want to determine which of these effects are important and which are not. There are several ways to determine this, including using standard hypothesis testing approaches. For this paper we opted not to use formal hypothesis tests primarily because they require strong assumptions about the standard deviation of the experimental samples. In future work we will avoid this problem by simply replicating observations in the experimental design. For this work however, we display the effects graphically and ask developers to use their expert judgement to decide which effects they consider important.

\section{Feasibility Study}

This section describes a feasibility study that assesses the implementation cost and the effectiveness of the main effects screening process described in Section 3 on a suite of large, performance-intensive software systems.

\subsection{Experimental Design}

Hypotheses. Our feasibility study explores the following hypotheses: (1) our model-based Skoll environment costeffectively supports the definition, implementation and execution of our main effects screening process described in Section 3, (2) the screening design used in main effects screening correctly identifies a small subset of options whose effect on performance is important, and (3) exhaustively examining just the options identified by the screening design gives performance data that (a) is representative of the system's performance across the entire configuration space, but less costly to obtain and (b) is more representative than a similarly-sized random sample.

Subject applications. The experimental subject applications for this study were based on a suite of performanceintensive software: ACE v5.4 + TAO v1.4 + CIAO v0.4. ACE provides reusable $\mathrm{C}++$ wrapper facades and framework components that implements core concurrency and distribution patterns [13] for communication software. TAO is a high-performance, highly configurable Real-time CORBA ORB built atop ACE to meet the demanding QoS requirements of DRE systems. CIAO is QoS-enabled middleware that extends TAO to support components, which enables developers to declaratively provision QoS policies end-to-end when assembling a DRE system.

ACE, TAO, and CIAO are ideal subjects for our feasibility study since they share many characteristics with other highly configurable performance-intensive software systems. For example, they collectively contain over $2 \mathrm{M}+$ lines of source code, functional regression tests, and performance benchmarks contained in $\sim 4,500$ files that average over 300 CVS commits per week. They also run on a wide range of OS platforms, including all variants of Windows, most versions of UNIX, and many real-time operating systems, such as LynxOS and VxWorks.

Application scenario. Due to recent changes made to the message queuing strategy, the developers of ACE+TAO+CIAO were concerned with measuring two performance criteria: (1) the latency for each request and (2) total message throughput (events/second) between the ACE+TAO+CIAO client and server. For this version of ACE+TAO+CIAO, the developers identified 14 binary run-time options they felt affected latency and throughput (See Table 2). Thus, the entire configuration space has $2^{14}=16,384$ different configurations.

Experimental process. Our experimental process used Skoll's model-driven tools to implement the main effects screening process and evaluate our three hypotheses. To do this, we executed the main effects screening process across a prototype Skoll grid of dual processor Xeon machines running Red Hat 2.4.21 with 1GB of memory in the real-time scheduling class. The experimental task involved running a benchmark application in a particular configuration, which evaluated the application scenario outlined above by creating an ACE+TAO+CIAO client and server. For each task we measured message latency and overall throughput between the client and the server. The client sends $300 \mathrm{~K}$ requests to 


\begin{tabular}{|c|l|c|}
\hline $\begin{array}{c}\text { Option } \\
\text { Index }\end{array}$ & Option & Option \\
Settings \\
\hline A & ORBReactorThreadQueue & $\{$ FIFO, LIFO $\}$ \\
B & ORBClientConnectionHandler & $\{$ RW, MT $\}$ \\
C & ORBReactorMaskSignals & $\{0,1\}$ \\
D & ORBConnectionPurgingStrategy & $\{$ LRU, LFU $\}$ \\
E & ORBConnectionCachePurgePercentage & $\{10,40\}$ \\
F & ORBConnectionCacheLock & $\{$ thread, null $\}$ \\
G & ORBCorbaObjectLock & $\{$ thread, null $\}$ \\
H & ORBObjectKeyTableLock & $\{$ thread, null $\}$ \\
I & ORBInputCDRAllocator & $\{$ thread, null $\}$ \\
J & ORBConcurrency & $\{32,128\}$ \\
K & ORBActiveObjectMapSize & reactive, thread-per-connection $\}$ \\
L & ORBUseridPolicyDemuxStrategy & $\{$ linear, dynamic $\}$ \\
M & ORBSystemidPolicyDemuxStrategy & $\{$ linear, dynamic $\}$ \\
N & ORBUniqueidPolicyReverseDemuxStrategy & \\
\hline
\end{tabular}

Table 2. Some ACE+TAO Options and their Settings

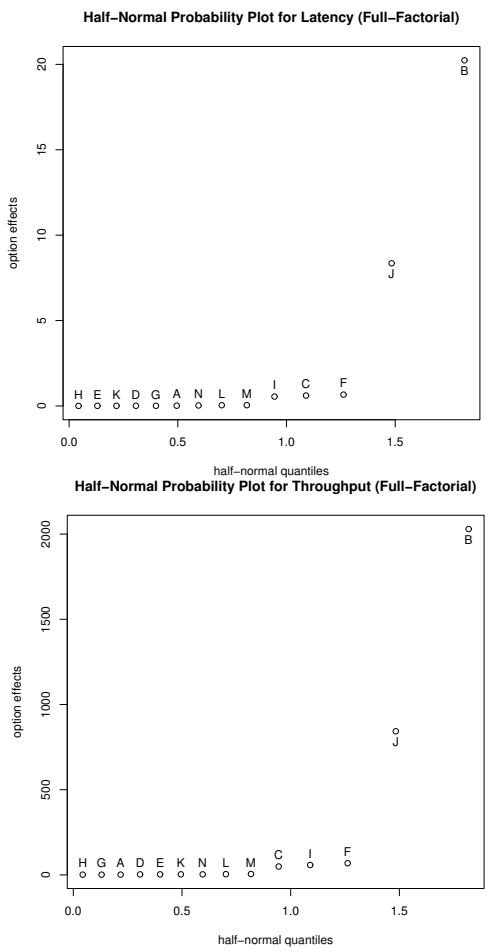

Figure 2. Option effects based on full data

the server, where after each request it waits for a response from the server and records the latency measure. At the end of $300 \mathrm{~K}$ requests, the client computes the throughput achieved in terms of number of requests served per second. We finally analyzed the resulting data to evaluate our hypotheses. Section 6 describes the limitations with our current experimental process.

\subsection{The Full Data Set}

To evaluate our approach, we generated performance data for all $16,000+$ valid configurations, which we refer to as the "full suite" and the performance data as the "full data set." We then examined the effect of each option and judged whether they had important effects on performance using a graphical method called half-normal probability plots, which show each option's effect against their corre- sponding coordinates on the half-normal probability scale. If $|\theta|_{1} \leq|\theta|_{2} \leq \ldots \leq|\theta|_{I}$ are the ordered set of effect estimations, the half-normal plot then consists of the points

$$
\left(\Phi^{-1}(0.5+0.5[i-0.5] / I),|\theta|_{i}\right) \text { for } i=1, \ldots, I
$$

where $\Phi$ is the cumulative distribution function of a standard normal random variable.

The rationale behind half-normal plots is that unimportant options will have effects whose distribution is normal and centered near 0 . Important effects will also be normally distributed with means different that 0 . If no effects are important, the resulting plot will show a set of points on a rough line near $y=0$. Options whose effects deviate substantially from 0 are considered important.

Note that "importance" is not defined formally and differs in spirit from the traditional notion of statistical significance. In particular, developers must decide for themselves how large effects must be to warrant their attention. While this has some downsides (see Section 6), even with traditional statistical tests that measure statistical significance developers still must make judgments as to the magnitude of effects.

Figure 2 plots the effect across the full data set of each of the 14 ACE+TA+CIAO options on latency and throughput. We see that options B and $\mathbf{J}$ are clearly important, whereas options I, C and F are arguably important, and the remaining options are not important.

\subsection{Evaluating Screening Designs}

We now evaluate whether the remotely executed screening designs can correctly identify the same important options discovered in the full data set. To do this, we calculated and executed three different screening designs, whose specifications appear in Appendix A. These designs examined all 14 options using increasingly larger run sizes (32, 64 , or 128 observations). We refer to the screening designs as $S c r_{32}, S c r_{64}$ and $S c r_{128}$, respectively.

Figure 3 shows the half-normal probability plots obtained from our screening designs. The figures show that all screening designs correctly identify options $\mathrm{B}$ and $\mathrm{J}$ as being important (as is the case in full-factorial experiment). $S c r_{128}$ also identifies the possibly important effect of options C, I, and F. Due to space considerations in the paper we only present data on latency. Throughput analysis shows identical results unless otherwise stated.

These results suggest that (1) screening designs can detect important options at a small fraction of the cost of exhaustive testing, (2) the smaller the effect, the larger the run size needed to identify it, and (3) developers should be cautious when dealing with options that appear to have an important, but relatively small effect, as they may actually be seeing normal variation $\left(\mathrm{Scr}_{32}\right.$ and $\mathrm{Scr}_{64}$ both have examples of this). 

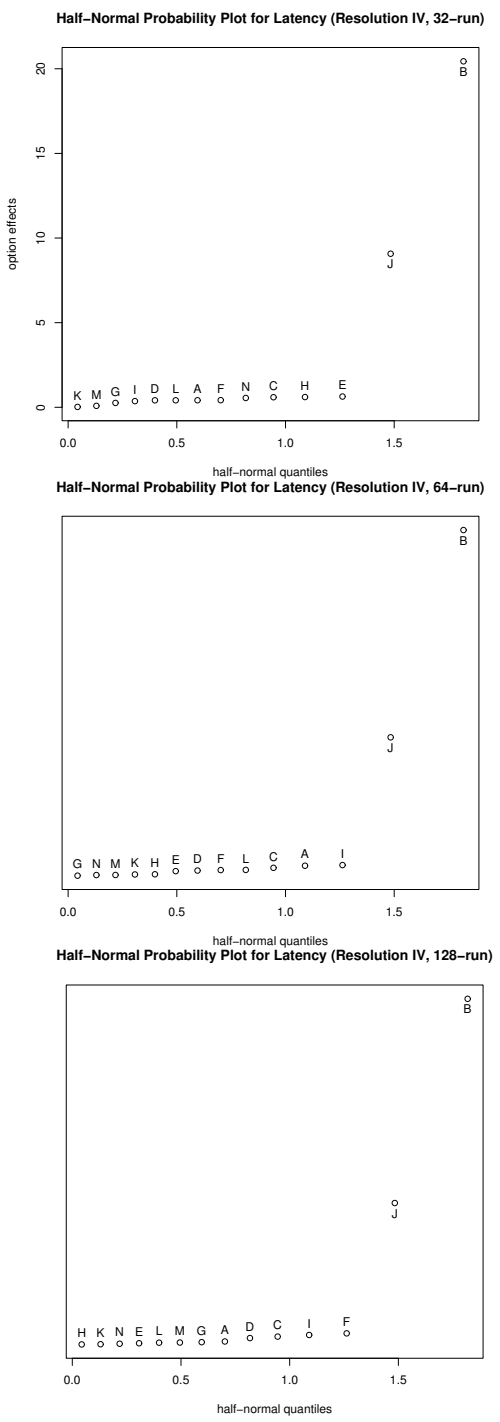

Figure 3. Option Effects Based on Screening Designs

\subsection{Estimating Performance with Screening Suites}

We now evaluate whether screening all the combinations of the most important options can be used to estimate performance quickly across the entire configuration space we are studying. The estimates are generated by examining all combinations of the most important options, while defaulting the settings of the unimportant options. In the previous section, we determined that options B and $\mathrm{J}$ were clearly important and that options $\mathrm{C}$, I, and F were arguably important. Developers will therefore make the estimates based on benchmarking either 4 (all combinations of options B and J) or 32 (all combinations of options B, J, C, I, and F) configurations. We will refer to the set of 4 configurations as the top- 2 screening suite and the set of 32 configurations as the top-5 screening suite.
Figure 4 shows the distributions of latency for the full suite vs. the top-5 screening suite and for the full suite vs. the top- 2 screening suite. From the figure, we see that the distributions of the top-5 and top- 2 screening suites closely track the overall performance data. Such plots, called quantile-quantile (Q-Q) plots, are used to see how well two data distributions correlate. To do this they plot the quantiles of the first data set against the quantiles of the second data set. If the two sets share the same distribution, the points should fall approximately on $x=y$ line. In addition we performed Mann-Whitney non-parametric tests to determine whether each set of screening data (top- 2 and top- 5 suites) appears to come from the same distribution as the full data. In both cases we were unable to reject the null hypothesis that the top- 2 and top- 5 screening suite data come from the same distribution as the full suite data. This data suggests that the screening suites computed at step 4 of the main effects screening process (Section 3 ) can be used to estimate overall performance in-house at extremely low time/effort, i.e., running 4 benchmarks takes 40 seconds, running 32 takes 5 minutes, running 16,000+ takes 2 days.

\subsection{Screening Suites vs. Random Sampling}
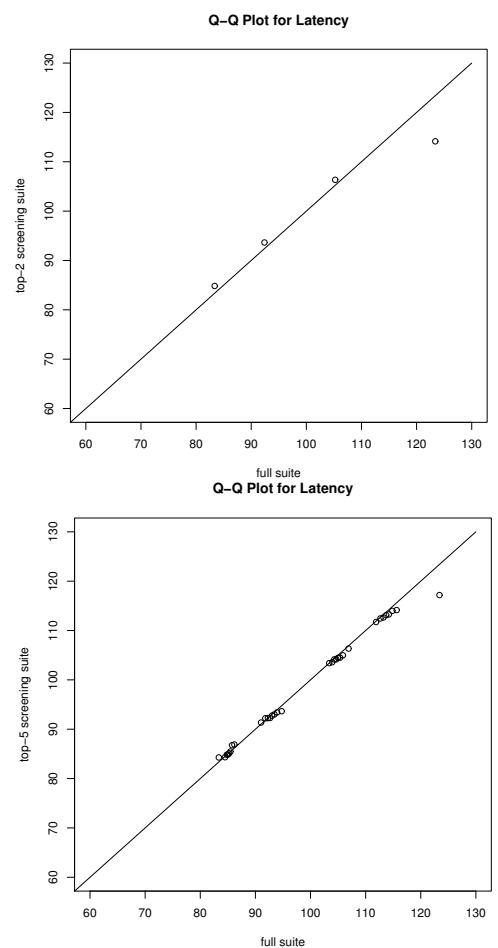

Figure 4. Q-Q plots for the top-2 and top-5 screening suites

Another question is whether our main effects screening process was any better than other low-cost estimation processes. In particular, we compared the latency distributions of several random samples of 4 configurations to that of 


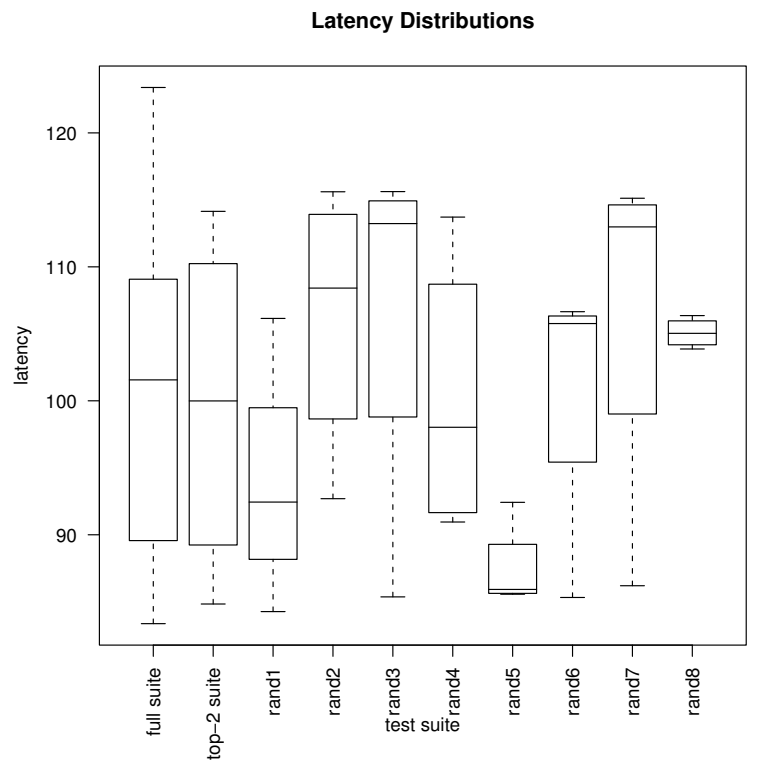

Figure 5. Latency Distribution from full, top-2, and random suites

the top- 2 screening suite found by our process. The results of this test are summarized in Figure 5. These box plots show the distributions of latency metric obtained from exhaustive testing, top- 2 screening suite testing, and random testing. These graphs suggest the obvious weakness of random sampling, i.e., while sampling distributions tend toward the overall distribution as the sample size grows, individual small samples may show wildly different distributions.

\subsection{Dealing with Evolving Systems}

A primary goal of main effects screening is to detect performance degradations in evolving systems quickly. So far we have not addressed whether - or for how long screening suites remain useful as a system evolves. To better understand this issue, we measured latency on the top-2 screening suite, once a day, using CVS snapshots of ACE+TAO+CIAO. We used historical snapshots for two reasons: (1) the versions are from the time period for which we already calculated the main effects and (2) developer testing and in-the-field usage data have already been collected and analyzed for this time period (see www.dre. vanderbilt.edu/Stats/), allowing us to assess the system's performance without having to exhaustively test all configurations for each system change.

Figure 6 depicts the data distributions for the top-2 screening suites broken down by date (higher latency measures are worse). We see that the distributions were stable the first two days, crept up somewhat for 3 days and then shot up the $4^{\text {th }}$ day (12/14/03). They were brought back under control for several more days, but then moved up again on the last day. Developer records and problem reports indi- cate that problems were noticed on 12/14/03, but not before then.

Another interesting finding was that the limited testing done by ACE+TAO+CIAO developers measured a performance drop of only around 5\% on 12/14/03. In contrast, our screening process showed a much more dramatic drop - closer to 50\%. Further analysis by system developers indicated that their unsystematic testing failed to evaluate configurations where the degradation was much more pronounced.

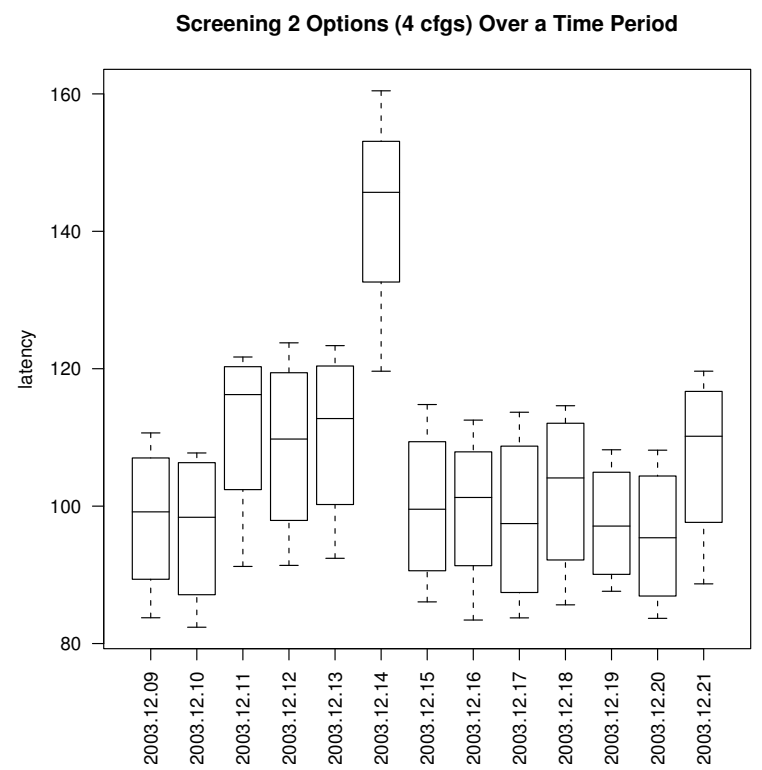

Figure 6. Performance estimates across time

\subsection{Higher-Order Effects}

The analyses done so far only calculated first-order effects, which worked well for our subject application and scenario, but might not be sufficient for other situations. Figure 7 shows the effects of all pairs of options in the subject systems based on the full data set and on a screening design. We used a resolution VI design here (rather than resolution IV as in the previous sections) and increased the run size to 2,048 to capture the second-order effects. From the figure we see several things. First, the important interaction effects involve only options that are already considered important by themselves, which supports the idea that monitoring only first-order effects was sufficient for our subject systems. Second, we see that the screening design correctly identifies the 5 most important pairwise interactions at $1 / 8^{\text {th }}$ the cost of exhaustive testing.

\section{Related Work}

Applying DOE to software engineering. As far as we can tell, no one has used screening designs for software performance assessment. The use of design of experiments (DoE) theory within software engineering has mostly been 

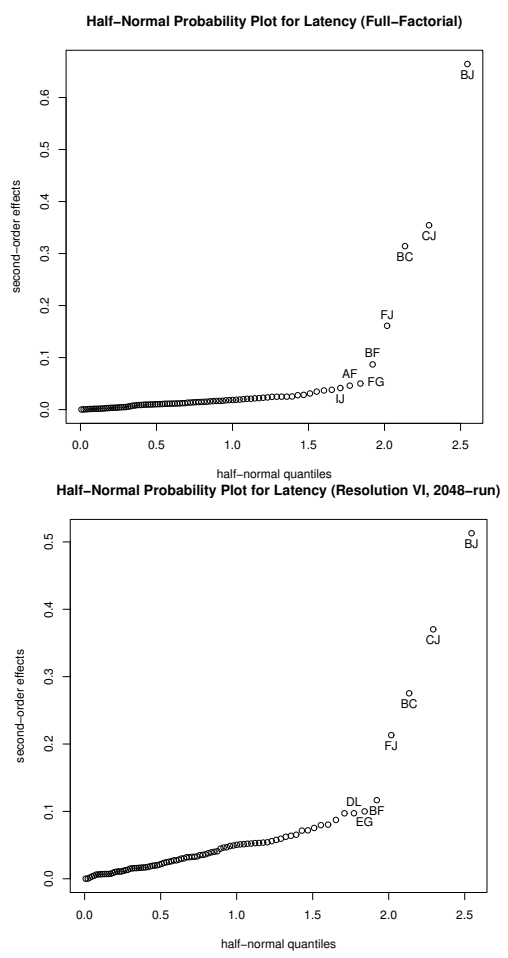

Figure 7. Pairwise Effects Based on Full and Screening Suite

limited to interaction testing, largely to compute and sometimes generate minimal test suites that cover all combinations of specified program inputs. Some examples of this work include Dalal et al. [4], Burr et al. [2], Dunietz et al. [5], and Kuhn et al [10]. Yilmaz et al. [16] used covering arrays as a configuration space sampling technique to support the characterization of failure-inducing option settings. Other relevant literature on performance monitoring includes:

- Offline analysis, which has been applied to program analysis to improve compiler-generated code. For example, the ATLAS [6] numerical algebra library uses an empirical optimization engine to decide the values of optimization parameters by generating different program versions that are run on various hardware/OS platforms. The output from these runs are used to select parameter values that provide the best performance. Mathematical models are also used to estimate optimization parameters based on the underlying architecture, though empirical data is not fed into the models to refine it.

- Online analysis, where feedback control is used to dynamically adapt QoS measures. An example of online analysis is the ControlWare middleware [17], which uses feedback control theory by analyzing the architecture and modeling it as a feedback control loop. Actuators and sensors then monitor the system and affect server resource al- location. Real-time scheduling based on feedback loops has also been applied to Real-time CORBA middleware [11] to automatically adjust the rate of remote operation invocation transparently to an application.

- Hybrid analysis, which combines aspects of offline and online analysis. For example, the continuous compilation strategy [3] constantly monitors and improves application code using code optimization techniques.

\section{Concluding Remarks}

This paper presents a new distributed continuous quality assurance (DCQA) process called main effects screening that is designed to detect performance degradation efficiently in performance-intensive software systems that have large configuration spaces. To evaluate this process, we conducted a formally-designed experiment across a grid of in-house and in-the-field computers in the Skoll environment. The results of this experiment helped in estimating performance across the large configuration space of ACE, TAO, and CIAO software systems.

All empirical studies suffer from threats to their internal and external validity. For this work, we were primarily concerned with threats to external validity since they limit our ability to generalize the results of our experiment to industrial practice. One potential threat is that several steps in our process require human decision making and input, e.g., developers must provide reasonable benchmarking applications and must also decide when they consider effects to be important.

Another possible threat to external validity concerns the representativeness of the ACE+TAO+CIAO subject applications, which though large are still just one suite of software systems. A related issue is that we have focused on a relatively simple and small subset of the entire configuration space of ACE+TAO+CIAO that only has binary options and has no inter-option constraints. While these issues pose no theoretical problems (screening designs can be created for much more complex situations), we need to apply our approach to larger, more realistic configuration spaces in future work to understand how well it scales.

Another potential threat is that for the time period we studied, the ACE+TAO+CIAO subject application was in a fairly stable phase, i.e., changes were made mostly to fix bugs and reduce memory footprint, but the system's functionality was relatively stable. For situations where a system's basic functionality is in greater flux, it may be harder to distinguish significant performance degradation from normal variation.

Despite these limitations, we believe our study supports our basic hypotheses. We reached this conclusion by noting that our studies showed that: (1) screening designs can correctly identify important options, (2) these options can be used to quickly produce reliable estimates of performance across the entire configuration space at a fraction of the cost 
of exhaustive testing, (3) the alternative approach of random or ad hoc sampling can give highly unreliable results, (4) the main effects process detected performance degradation on a large and evolving software system, and (5) the screening suite estimates were more precise than the ad hoc process currently used by the developers of the subject system. Main effects screening can also be a defect detection aid, e.g., if the screened options change unexpectedly, developers can reexamine the software to identify possible problems with software updates.

We believe that this line of research is novel and interesting, but much work remains to be done. We are therefore continuing to develop enhanced model-based Skoll capabilities and using them to create and validate new more sophisticated DCQA processes that overcome existing limitations and threats to external validity. In particular, we are exploring the connection between design of experiments theory and the quality assurance of systems with large configuration spaces. We are also working to incorporate Skoll services into software repositories, such as ESCHER (www. escherinstitute. org). Finally, we are conducting a much larger case study using Skoll to conduct the ACE+TAO+CIAO daily build and regression test process with 100+ machines contributed by users and developers worldwide.

Acknowledgements We thank the anonymous reviewers for their helpful comments. This material is based on work supported by the US National Science Foundation under NSF grants CCR-0312859, CCR- 0205265, CCR-0098158 and CCF-0447864 as well as funding from BBN Technologies, Lockheed Martin, Raytheon, and Siemens.

\section{References}

[1] L. Breiman, J. Freidman, R. Olshen, and C. Stone. Classification and Regression Trees. Wadsworth, Monterey, CA, 1984.

[2] K. Burr and W. Young. Combinatorial test techniques: Table-based automation, test generation and code coverage. In Proc. of the Intl. Conf. on Software Testing Analysis \& Review, 1998.

[3] B. Childers, J. Davidson, and M. Soffa. Continuous Compilation: A New Approach to Aggressive and Adaptive Code Transformation. In Proceedings of the International Parallel and Distributed Processing Symposium, Apr. 2003.

[4] S. R. Dalal, A. Jain, N. Karunanithi, J. M. Leaton, C. M. Lott, G. C. Patton, and B. M. Horowitz. Model-based testing in practice. In Proc. of the Intl. Conf. on Software Engineering, (ICSE), pages 285-294, 1999.

[5] I. S. Dunietz, W. K. Ehrlich, B. D. Szablak, C. L. Mallows, and A. Iannino. Applying design of experiments to software testing. In Proc. of the Intl. Conf. on Software Engineering, (ICSE '97), pages 205-215, 1997.

[6] Kamen Yotov and Xiaoming Li and Gan Ren et.al. A Comparison of Empirical and Model-driven Optimization. In Proceedings of ACM SIGPLAN conference on Programming Language Design and Implementation, June 2003.
[7] G. Karsai, J. Sztipanovits, A. Ledeczi, and T. Bapty. ModelIntegrated Development of Embedded Software. Proceedings of the IEEE, 91(1):145-164, Jan. 2003.

[8] A. S. Krishna, D. C. Schmidt, A. Porter, A. Memon, and D. Sevilla-Ruiz. Improving the Quality of Performanceintensive Software via Model-integrated Distributed Continuous Quality Assurance. In Proceedings of the 8th International Conference on Software Reuse, Madrid, Spain, July 2004. ACM/IEEE.

[9] A. S. Krishna, N. Wang, B. Natarajan, A. Gokhale, D. C. Schmidt, and G. Thaker. CCMPerf: A Benchmarking Tool for CORBA Component Model Implementations. In Proceedings of the 10th Real-time Technology and Application Symposium (RTAS '04), Toronto, CA, May 2004. IEEE.

[10] D. Kuhn and M. Reilly. An investigation of the applicability of design of experiments to software testing. Proc. 27th Annual NASA Goddard/IEEE Software Engineering Workshop, pages 91-95, 2002.

[11] C. Lu, J. A. Stankovic, G. Tao, and S. H. Son. Feedback Control Real-Time Scheduling: Framework, Modeling, and Algorithms. Real-Time Systems Journal, 23(1/2):85-126, July 2002.

[12] A. Memon, A. Porter, C. Yilmaz, A. Nagarajan, D. C. Schmidt, and B. Natarajan. Skoll: Distributed Continuous Quality Assurance. In Proceedings of the 26th IEEE/ACM International Conference on Software Engineering, Edinburgh, Scotland, May 2004. IEEE/ACM.

[13] D. C. Schmidt, M. Stal, H. Rohnert, and F. Buschmann. Pattern-Oriented Software Architecture: Patterns for Concurrent and Networked Objects, Volume 2. Wiley \& Sons, New York, 2000.

[14] E. Turkay, A. Gokhale, and B. Natarajan. Addressing the Middleware Configuration Challenges using Model-based Techniques. In Proceedings of the 42nd Annual Southeast Conference, Huntsville, AL, Apr. 2004. ACM.

[15] C. F. J. Wu and M. Hamada. Experiments: Planning, Analysis, and Parameter Design Optimization. Wiley, 2000.

[16] C. Yilmaz, M. B. Cohen, and A. Porter. Covering arrays for efficient fault characterizations in complex configuration space. In Proceedings of the ACM SIGSOFT International Symposium on Software Testing and Analysis (ISSTA), 2004.

[17] R. Zhang, C. Lu, T. Abdelzaher, and J. Stankovic. Controlware: A Middleware Architecture for Feedback Control of Software Performance. In Proceedings of the International Conference on Distributed Systems 2002, July 2002.

\section{A. Actual Screening Designs}

The screening designs used in Section 4.3 were calculated using the SAS statistical package. (WWw. sas. com). $S c r_{3} 2$ is a $2_{I V}^{14-9}$ with design generators $F=A B C, G=$ $A B D, H=A C D, I=B C D, J=A B E, K=A C E$, $L=B C E, M=A D E, N=B D E$.

$S c r_{6} 4$ is a $2_{I V}^{14-8}$ with design generators $G=A B C, H=$ $A B D, I=A B E, J=A C D E, K=A B F, L=A C D F$, $M=A C E F, N=A D E F$.

$S c r_{1} 28$ is a $2_{I V}^{14-7}$ with design generators $H=A B C, I=$ $A B D E, J=A B D F, K=A C E F, L=A C D G, M=$ $A B E F G, N=B C D E F G$. 